\title{
Targeted Gene Expression in Zebrafish Exposed to Chlorpyrifos- Oxon Confirms Phenotype-Specific Mechanisms Leading to Adverse Outcomes
}

\author{
Natàlia Garcia-Reyero ${ }^{1,2} \cdot$ Lynn Escalon $^{1} \cdot$ Eva Prats $^{3} \cdot$ Melissa Faria $^{4,5}$ • \\ Amadeu M. V. M. Soares ${ }^{4}$. Demetrio Raldúa ${ }^{5}$
}

Received: 10 January 2016/ Accepted: 7 April 2016/Published online: 16 April 2016

(C) The Author(s) 2016. This article is published with open access at Springerlink.com

\begin{abstract}
Zebrafish models for mild, moderate, and severe acute organophosphorus poisoning were previously developed by exposing zebrafish larvae to chlopyrifosoxon. The phenotype of these models was characterized at several levels of biological organization. Oxidative stress and mitochondrial dysfunction were found to be involved in the development of the more severe phenotype. Here we used targeted gene expression to understand the dose-responsiveness of those two pathways and their involvement on generating the different zebrafish models. As the severe phenotype is irreversible after only $3 \mathrm{~h}$ of exposure, we also analyzed the response of the oxidative stress pathway at 3 and $24 \mathrm{~h}$. Some of the genes related to oxidative stress were already differentially expressed at $3 \mathrm{~h}$. There was an increase in differentially expressed genes related to both oxidative stress and mitochondrial function from the more mild to the more severe phenotype, suggesting the involvement of these mechanisms in increasing phenotype severity. Temporal data suggest that peroxynitrite leading to lipid peroxidation might be involved in phenotype transition and irreversibility.
\end{abstract}

Natàlia Garcia-Reyero

natalia.g.vinas@erdc.dren.mil

1 US Army Engineer Research and Development Center, 3909 Halls Ferry Rd, Vicksburg, MS 39180, USA

2 Institute for Genomics, Biocomputing \& Biotechnology, Mississippi State University, 2 Research Blvd, Starkville, MS 39759, USA

3 CIC-CSIC, Jordi Girona 18, 08034 Barcelona, Spain

4 Department of Biology and CESAM, University of Aveiro, Aveiro, Portugal

5 IDAEA-CSIC, Jordi Girona 18, 08034 Barcelona, Spain
Keywords Zebrafish - Gene expression - Oxidative stress $\cdot$ Mitochondria $\cdot$ Chlorpyrifos-oxon

Organophosphorus compounds (OP) are acetylcholinesterase (AChE) inhibitors used primarily in pest control. There are over 3 million cases of severe acute OP poisoning (OPP) reported annually, resulting in 300,000 deaths (Bertolote 2006). Exposure to OP inhibits AChE activity leading to the accumulation of the neurotransmitter acetylcholine (ACh), activation of ACh receptors, and overstimulation of cholinergic neurons and seizures. This leads to the release of excitatory amino acids. Those will activate $N$-methyl-D-aspartate (NMDA) receptors. This results in an intracellular influx of $\mathrm{Ca}^{2+}$, which can cause cell damage, necrosis, and apoptosis (Brookes 2004; PenaLlopis 2005; Kaur et al. 2014).

Organophosphate exposure has also been linked to honey bee and Daphnia mortality, toxicity in fish, deterioration of macroinvertebrate communities, and potential reduction in fish population growth (Southam et al. 2011; Echeverría-Sáenz et al. 2012; Zhu et al. 2014; Macneale et al. 2014; Calatayud-Vernich et al. 2016). It is therefore important to better understand the effects and mechanisms of action of OPP to better estimate hazards to organisms and populations.

A zebrafish (Danio rerio) model for mild, moderate, and severe OP poisoning has been previously documented (Faria et al. 2015). In order to develop the model, zebrafish larvae were exposed to increasing concentrations of chlorpyrifos-oxon (CPO), the active metabolite of the pesticide chlorpyrifos and a prototypic OP compound. The mild (P1) phenotype was characterized by behavioral impairment, which correlated with AChE inhibition. The moderate phenotype (P2) exhibited hypercontracture of the 
axial muscle fibers. The severe phenotype (P3), irreversible after only $3 \mathrm{~h}$ of exposure to CPO, was characterized by necrosis at the central nervous and neuromuscular systems. Interestingly, $\mathrm{P} 3$ was the only phenotype that presented a dysregulation of $\mathrm{Ca}^{2+}$ homeostasis, oxidative stress, and disruption of mitochondrial structure and function (Faria et al. 2015).

In order to understand the changes that triggered the transition from one phenotype to the next, we analyzed expression changes from a set of genes involved in mitochondrial function and oxidative stress in all three phenotypes. We also explored the expression of oxidative stressrelated genes at 3 and $24 \mathrm{~h}$ on P3 embryos to explore the potential changes leading to the irreversibility of that phenotype.

\section{Materials and Methods}

Exposures Embryos from wild-type zebrafish were obtained by natural mating and maintained in fish water at $28.5^{\circ} \mathrm{C}$. Larvae were not fed during the entire experimental period. All procedures were conducted in accordance with institutional guidelines and approved by the Institutional Animal Care and Use Committee at the Research and Development Centre of the Spanish Research Council (CID-CSIC). The stability of CPO (Chem Service, $98.1 \%$ purity) in fish water under exposure conditions was tested by LC-MS/MS and has been published elsewhere (Faria et al. 2015). Briefly, 7 days post-fertilization (dpf) zebrafish larvae were exposed to nominal concentrations of 0.1 , 1 , and $3 \mu \mathrm{M}$ CPO in fish water, with $\mathrm{n}=3$ replicates per treatment. Control larvae were exposed under identical conditions to the same concentration of the carrier $(0.1 \%$ DMSO). Water samples were collected at 0 and $24 \mathrm{~h}$ of exposure and immediately analyzed using a Luna C18 $(150 \mathrm{~mm} \times 2 \mathrm{~mm} \mathrm{ID}$, particle size $5 \mu \mathrm{m}$, Phenomenex, Torrance, CA) equipped with a Security Guard pre-column. Measured CPO concentrations in water were $0,0.097$, 0.995 , and $2.99 \mu \mathrm{M}$ at $0 \mathrm{~h}$ of exposure and $0,0.038,0.684$, and $1.99 \mu \mathrm{M}$ after $24 \mathrm{~h}$ of exposure. Hydrolysis of CPO in water has been extensively studied, with a half-life dependent on the initial concentration (Jacobson et al. 2010). The uptake of CPO would also be responsible of a decrease of water concentration. Criteria used to classify the severity of OPP in zebrafish larvae has already been published elsewhere (Faria et al. 2015).

RNA Extraction Total RNA was isolated from pools of 5 larvae after thorough homogenization using a NucleoSpin RNA XS kit (Macherey-Nagel, GmbH \& Co. KG, Düren, Germany) following the manufacturer's recommendations. RNA quantity and quality were analyzed using a Bioanalyzer (Agilent Technologies, Santa Clara, CA) and a
Nanodrop ND-1000 spectrophotometer (Nanodrop Technologies, Wilmington, DE). Only RNA considered to be of good quality (RIN $>8$ ) was used for real-time PCR analysis.

Real-Time PCR The RT2 Profiler Zebrafish PCR Arrays for genes related to mitochondria (catalog number PAZF087Z) and oxidative stress (catalog number PAZF-065Z) were obtained from Qiagen (Valencia, CA) in a 384-well format, which included 4 replicates of 84 genes plus standard controls (housekeeping genes, reverse transcription controls and positive PCR controls). Four biological replicates were used per treatment. The real-time PCR (RT-PCR) assays were performed on an ABI Sequence Detector 7900 (Applied Biosystems, Foster City, CA). Briefly, a total of $5 \mu \mathrm{g}$ RNA was used to synthesize cDNA with the RT2 First Strand Kit (Qiagen, Valencia, CA) following the manufacturer's protocol. The RT2 SYBR Green Mastermix (Qiagen, Valencia, CA) was used for RTPCR, following the Qiagen protocol for 384 well plates containing 4 replicates of 96 assays. Cycling parameters were $95^{\circ} \mathrm{C}$ for $10 \mathrm{~min}, 40$ cycles of $95^{\circ} \mathrm{C}$ for $15 \mathrm{~s}$ and $60^{\circ} \mathrm{C}$ for $1 \mathrm{~min}$. The GeneGlobe Data Analysis Center (Qiagen, Valencia, USA) was used to analyze the data using the $\Delta \Delta \mathrm{Ct}$ method $(p<0.05)$ with normalization of the raw data to 5 different housekeeping genes. Only differentially expressed genes are included in the results.

\section{Results and Discussion}

The NMDA receptors are ion channels activated by excitatory amino acids after their release due to OP-induced seizures. In neurons, mitochondria localized in proximity of the NMDA receptors, accumulate $\mathrm{Ca}^{2+}$ and prevent the spreading of a cytosolic wave (Rizzuto et al. 2012) (Fig. 1). The continuous activation of these receptors will lead to increased intracellular calcium, which will eventually result in mitochondrial membrane depolarization, dysregulation of mitochondrial $\mathrm{Ca}^{2+}$ homeostasis, production of reactive nitrogen and oxygen species (RNS and ROS), and cellular toxicity (Dong et al. 2009).

Mitochondria are the center of cellular energy metabolism, where most ATP synthesis occurs stimulated by $\mathrm{Ca}^{2+}$, a key regulator of mitochondrial function. Dysregulation of mitochondrial $\mathrm{Ca}^{2+}$ homeostasis can lead to the generation of ROS, triggering the permeability transition pore (PTP), cytochrome $\mathrm{c}$ release, and eventually apoptosis (Brookes 2004). The release of cytochrome c begins with its dissociation from its binding site, which increases its level in the intermembrane space. Cytochrome $\mathrm{c}$ is subsequently released by pore formation mediated by the proapoptotic Bcl-2 proteins, which eventually leads to apoptosis (Orrenius et al. 2007). The intracellular calcium 


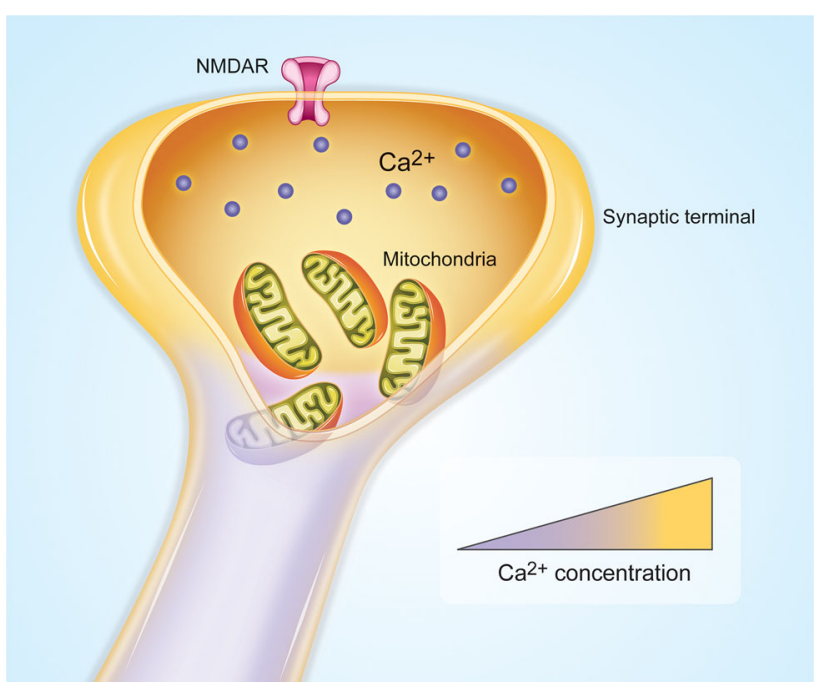

Fig. 1 In neurons, mitochondria localized in proximity of $\mathrm{Ca}^{2+}$ channels such as NMDA receptors (NMDAR) accumulate $\mathrm{Ca}^{2+}$ and prevent the spreading of a cytosolic wave. From Rizzuto et al. (2012)

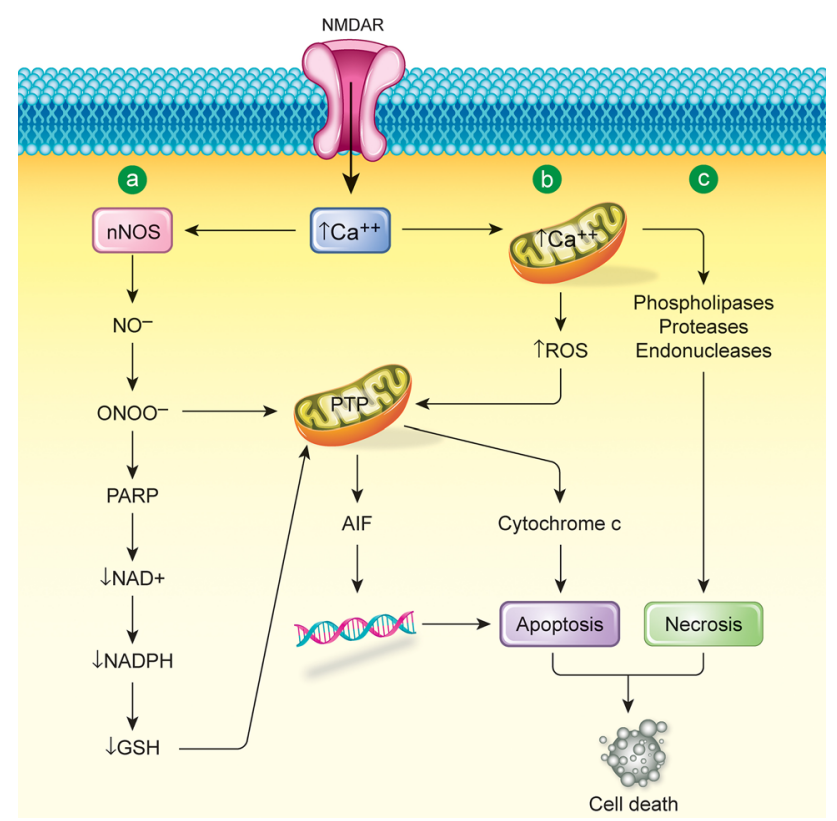

Fig. 2 Activation of $N$-methyl-D-aspartate (NMDA) receptors can result in an intracellular influx of $\mathrm{Ca}^{3+}$, which can lead to $a$ RNS, peroxy nitrite, $b$ ROS production leading to apoptosis, $c$ necrosis, and eventually cell death. From Kritis et al. (2015)

overload can also result in stimulation of $\mathrm{Ca}^{2+}$-dependent catabolic enzymes, such as phospholipases, proteases and endonucleases that will induce necrosis (Orrenius et al. 2015) (Fig. 2).

In addition, the activation of NMDA receptors and the increase of $\mathrm{Ca}^{2+}$ influx lead to the production of nitric oxide levels through the activation of the nitric oxide synthase (NOS) (Kritis et al. 2015). Elevated nitric oxide
(NO) concentrations can result in the production of the oxidant species peroxynitrite anion $\left(\mathrm{ONOO}^{-}\right)$, which causes lipid peroxidation, persistent inhibition of cytochrome c oxidase, and DNA damage in neurons leading to poly(ADP-ribose) polymerase -1 (Parp1) activation. The activation of Parp1 however, only contributes to damage when it is sufficient to significantly deplete $\mathrm{NAD}^{+}$(Moncada and Bolanos 2006).

As reported earlier, P3 was characterized by widespread necrosis, generation of ROS, dysregulation of the antioxidant defense system, lipid peroxidation, and mitochondrial damage including strong reduction of mitochondrial respiration. None of those effects were measurable in either P1 or P2 phenotypes (Faria et al. 2015). By exploring the regulation of genes related to oxidative stress and mitochondrial function in all phenotypes, we aimed to understand the mechanisms that triggered the transition to the more adverse phenotype.

A total of 50 genes related to oxidative stress (Tables 1 and 2) and 60 related to mitochondrial function (Table 3) were tested by RT-PCR in all three phenotypes. Unannotated genes or genes that did not pass the Qiagen standard quality control were removed from the analysis. In agreement with the phenotype severity gradient, P3 had the highest number of differentially expressed genes, followed by $\mathrm{P} 2$, and finally P1 had the lowest number of changes. Interestingly, most of the genes were up-regulated, particularly at P3, suggesting that somehow the organisms might have been trying to recover mitochondrial function.

Oxidative Stress The enzymes nos $2 a$ and noxal, both involved in the formation of peroxynitrite, were upregulated in P3. This result is consistent with the presence of lipid peroxidation, increased reactive nitrogen (RN) levels, and decreased GSH levels. Interestingly, nos $2 b$ was downregulated only in P2 and unchanged in P3. The noxal was upregulated in all three phenotypes, suggesting that recovery mechanisms were sufficient in P1 and P2 to deal with oxidative stress. Furthermore, the data suggests that the peroxynitrite route (Fig. 2a) was not significantly active in $\mathrm{P} 1$ and $\mathrm{P} 2$, as no presence of lipid peroxidation was found and only a slight decrease in GSH was detected in P2. Peroxyredoxins (Prdx) reduce peroxynitrite to nitrite (Trujillo et al. 2008). Prdx1 in particular, upregulated in P3, has been shown to inhibit oxidative stress induced apoptosis (Mei et al. 2015), which could be consistent with the need to reduce routes a and b (Fig. 2) in P3. In further support of these observations, three peroxyredoxins were significantly altered in $\mathrm{P} 3$, two in $\mathrm{P} 2$, and none in $\mathrm{P} 1$. Catalase (cat) and superoxide dismutase (sodl), part of the antioxidant defense activity, were downregulated in P2 and P3. Several gluthathione peroxidases and transferases were significantly altered in $\mathrm{P} 2$ and $\mathrm{P} 3$, but not P1.Thioredoxin $(t x n)$, an enzyme involved in decreasing oxidative stress, 
Table 1 RT-PCR results for the P1 (Grade 1), P2 (Grade 2) and P3 (Grade 3) embryos at $24 \mathrm{~h}$ for genes related to oxidative stress (Color table online)

\begin{tabular}{|c|c|c|c|c|c|c|c|c|c|}
\hline Symbol & P1 FC & P2 FC & P3 FC & Functional Grouping & Symbol & P1 FC & P2 FC & P3 FC & Functional Grouping \\
\hline gstk1 & - & -3.8767 & -4.4167 & Glutathione peroxidases & gclm & - & 2.6763 & 2.5867 & Oxidative stress responsive genes \\
\hline gstp1 & - & -2.3708 & -3.0783 & Glutathione peroxidases & $g p \times 1 b$ & - & 2.5417 & 4.3495 & Oxidative stress responsive genes \\
\hline gstz1 & - & - & -2.9757 & Glutathione peroxidases & gpx4a & - & -4.1066 & -4.3121 & Oxidative stress responsive genes \\
\hline apoea & - & -7.0465 & -6.5031 & Other antioxidants & $g p \times 7$ & - & -2.4585 & -2.1279 & Oxidative stress responsive genes \\
\hline vimp & -2.3928 & - & 2.0139 & Other antioxidants & gpx8 & - & -2.8839 & - & Oxidative stress responsive genes \\
\hline cybb & - & - & 2.5664 & Other peroxidases & hbl1 & -2.1133 & -2.609 & - & Oxidative stress responsive genes \\
\hline mgst3 & - & -3.9443 & -2.608 & Other peroxidases & hmox1a & -2.225 & $\cdot$ & 2.0034 & Oxidative stress responsive genes \\
\hline $\mathrm{mpx}$ & - & - & 3.1941 & Other peroxidases & hsp70 & - & $\cdot$ & 24.7488 & Oxidative stress responsive genes \\
\hline ptgs 1 & - & - & 2.1016 & Other peroxidases & hsp90aa 1.2 & - & 4.7813 & 14.7886 & Oxidative stress responsive genes \\
\hline ptgs $2 b$ & -4.3937 & - & 3.7852 & Other peroxidases & nqo1 & - & - & -2.1119 & Oxidative stress responsive genes \\
\hline aox1 & - & -2.9208 & -2.5754 & Other ROS metabolism genes & nudt1 & - & - & 2.4575 & Oxidative stress responsive genes \\
\hline bnip3 & - & - & -2.3317 & Other ROS metabolism genes & oxsr1b & - & - & -2.568 & Oxidative stress responsive genes \\
\hline ncf1 & - & 2.3232 & 4.673 & Other superoxide metabolism genes & pdlim1 & - & $\cdot$ & 2.8576 & Oxidative stress responsive genes \\
\hline nos2a & - & - & 2.47 & Other superoxide metabolism genes & sepp1a & - & -2.397 & -3.2891 & Oxidative stress responsive genes \\
\hline nos $2 b$ & - & -2.3326 & - & Other superoxide metabolism genes & sqstm1 & - & 4.1226 & 8.3472 & Oxidative stress responsive genes \\
\hline nox5 & - & -2.0082 & - & Other superoxide metabolism genes & srxn1 & - & -2.2655 & - & Oxidative stress responsive genes \\
\hline noxa1 & 2.7915 & 6.3904 & 15.7197 & Other superoxide metabolism genes & ttna & -2.347 & -3.6274 & - & Oxidative stress responsive genes \\
\hline ucp2 & $\cdot$ & 2.5412 & 3.0327 & Other superoxide metabolism genes & $\mathrm{txn}$ & $\cdot$ & 8.5462 & 14.1376 & Oxidative stress responsive genes \\
\hline bag2 & - & - & 2.7317 & Oxidative stress responsive genes & txnrd1 & - & 2.3858 & 2.3432 & Oxidative stress responsive genes \\
\hline cat & - & -2.4771 & -6.3711 & Oxidative stress responsive genes & cygb2 & - & - & -9.1058 & Oxygen transporters \\
\hline duox & -2.6379 & -2.4441 & - & Oxidative stress responsive genes & $\mathrm{mb}$ & -2.0431 & - & - & Oxygen transporters \\
\hline dusp1 & -3.2705 & - & - & Oxidative stress responsive genes & prdx1 & - & - & 2.122 & Peroxiredoxins \\
\hline fhl2a & - & -2.0772 & - & Oxidative stress responsive genes & prd $\times 2$ & - & -3.2326 & -2.6143 & Peroxiredoxins \\
\hline foxm1 & - & $\cdot$ & -2.03 & Oxidative stress responsive genes & prdx4 & - & -2.2773 & -2.828 & Peroxiredoxins \\
\hline fth1b & - & -2.5723 & -3.882 & Oxidative stress responsive genes & sod1 & - & -2.6303 & -2.6518 & Superoxide dismutase \\
\hline
\end{tabular}

Results are expressed as fold change (FC). Significantly expressed genes $(p<0.05)$ are highlighted in red (up-regulated), or blue (downregulated). Unchanged genes are highlighted in yellow. Gene list: aldehyde oxidase (aox), apolipoprotein Ea (apoea), BCL2-associated athanogene 2 (bag2), BCL2/adenovirus E1B interacting protein 3 (bnip3), catalase (cat), cytochrome b-245, beta polypeptide (cybb), cytoglobin 2 (cygb2), dual oxidase (duox), dual specificity phosphatase 1 (dusp1), forkhead box M1 (foxm1), ferritin heavy polypeptide 1a (fth1b), glutamatecysteine ligase modifier subunit $(\mathrm{gclm})$, glutathione peroxidase 1a $(\mathrm{gpx} 1 \mathrm{a})$, glutathione peroxidase $1 \mathrm{~b}(\mathrm{gpx} / \mathrm{b})$, glutathione peroxidase 4a $(\mathrm{gpx} 4 a)$, glutathione peroxidase 7 (gpx7), glutathione peroxidase 8 (gpx8), glutathione S-transferase kappa 1 (gstk1), glutathione S-transferase pi 1 (gstp1), glutathione S-transferase zeta 1 (gstzl), heme oxygenase (decycling) 1 ( $h m o x 1 a)$, heat shock cognate 70-kd protein ( $h s p 70)$, heat shock protein 90-alpha 2 (hsp90aal.2), myoglobin $(\mathrm{mb})$, microsomal gluthatione S-transferase 3 (mgst3), myeloid-specific peroxidase ( $m p x$ ), neutrophil cytosolic factor 1 ( $n c f 1$ ), nitric oxide synthase 2a (nos2a), NADPH oxidase activator 1 (noxa1), NAD(P)H dehydrogenase quinone 1 (nqo1), nudix (nucleoside diphosphate linked moiety X)-type motif 1 (nudt1), oxidative-stress responsive 1b (oxsr1b), PDZ and LIM domain 1 (elfin) (pdlim1), peroxiredoxin $1(\operatorname{prd} d x)$, peroxiredoxin $2(\operatorname{prd} d 2)$, peroxiredoxin $4(\operatorname{prd} x 4)$, prostaglandin-endoperoxide synthase 1 (ptgs1), prostaglandinendoperoxide synthase $2 \mathrm{~b}$ (ptgs $2 b$ ), selenoprotein P plasma 1a (seppla), superoxide dismutase 1 (sod1), sequestosome 1 (sqstm1), thioredoxin $(t x n)$, thioredoxin reductase 1 (txnrd1), uncoupling protein 2 (ucp2), VCP-interacting membrane selenoprotein (vimp)

was upregulated in P2 and P3. Necrosis (Fig. 2c) was also involved in P3 development, as shown in Faria et al. (2015).

As the more severe phenotype (P3) is reversible before $3 \mathrm{~h}$ of exposure, we analyzed genes relative to oxidative stress at both 3 and $24 \mathrm{~h}$ of exposure, in order to explore the initiating mechanisms that might lead to the permanent adverse outcome. Interestingly, nos $2 a$ and noxal were two of the few genes significantly up-regulated at $3 \mathrm{~h}$ (Table 2), suggesting that the peroxynitrite route (a) might be one of the initial mechanisms leading to toxicity in the cell. Two glutathione peroxidases, as well as $t x n$, were also up-regulated at the early time point, suggesting that the processes resulting in the formation of oxidative stress had already began. In addition, $h s p 70$ and $h s p 90 a a 1.2$, proteins that respond to stress, were already up-regulated at $3 \mathrm{~h}$.

Mitochondrial Function Twenty-six genes related to apoptosis and mitochondrial function were significantly 
Table 2 RT-PCR results for the P3 (Grade 3) embryos at 3 and $24 \mathrm{~h}$ for genes related to oxidative stress (Color table online)

\begin{tabular}{|c|c|c|c|c|c|c|c|}
\hline Gene Symbol & P3_3h FC & P3_24h FC & Functional Grouping & Gene Symbol & P3_3h FC & P3_24h FC & Functional Grouping \\
\hline gstk1 & - & -4.4167 & Glutathione Peroxidase & $g p \times 1 b$ & 6.1454 & 4.3495 & Oxidative stress responsive gene \\
\hline gstp1 & - & -3.0783 & Glutathione Peroxidase & $g p \times 4 a$ & - & -4.3121 & Oxidative stress responsive gene \\
\hline gstz1 & - & -2.9757 & Glutathione Peroxidase & gpx7 & - & -2.1279 & Oxidative stress responsive gene \\
\hline cybb & - & 2.5664 & Other Peroxidases & gpx8 & -2.7551 & - & Oxidative stress responsive gene \\
\hline cygb2 & - & -9.1058 & Other Peroxidases & hmox1a & - & 2.0034 & Oxidative stress responsive gene \\
\hline mgst3 & - & -2.608 & Other Peroxidases & hsp70 & 38.8869 & 24.7488 & Oxidative stress responsive gene \\
\hline $\mathrm{mpx}$ & $\cdot$ & 3.1941 & Other Peroxidases & hsp90aa 1.2 & 5.1633 & 14.7886 & Oxidative stress responsive gene \\
\hline ptgs1 & - & 2.1016 & Other Peroxidases & nqo1 & $\cdot$ & -2.1119 & Oxidative stress responsive gene \\
\hline ptgs $2 b$ & 2.6895 & 3.7852 & Other Peroxidases & nudt1 & - & 2.4575 & Oxidative stress responsive gene \\
\hline ncf1 & 4.0895 & 4.673 & Other Superoxide metabolism genes & oxsr1b & - & -2.568 & Oxidative stress responsive gene \\
\hline nos $2 a$ & 2.1964 & 2.47 & Other Superoxide metabolism genes & pdlim1 & 2.8951 & 2.8576 & Oxidative stress responsive gene \\
\hline noxa1 & 4.9337 & 15.7197 & Other Superoxide metabolism genes & sepp1a & - & -3.2891 & Oxidative stress responsive gene \\
\hline ucp2 & 3.3353 & 3.0327 & Other Superoxide metabolism genes & spstm1 & 3.7647 & 8.3472 & Oxidative stress responsive gene \\
\hline apoea & $\cdot$ & -6.5031 & Oxidative stress responsive gene & $\operatorname{txn}$ & 2.013 & 14.1376 & Oxidative stress responsive gene \\
\hline bag2 & - & 2.7317 & Oxidative stress responsive gene & txnrd1 & - & 2.3432 & Oxidative stress responsive gene \\
\hline cat & - & -6.3711 & Oxidative stress responsive gene & vimp & - & 2.0139 & Oxidative stress responsive gene \\
\hline duox & - & - & Oxidative stress responsive gene & $\mathrm{mb}$ & 2.5309 & - & Oxygen transporters \\
\hline dusp1 & 2.9644 & $\cdot$ & Oxidative stress responsive gene & prdx1 & - & 2.122 & Peroxiredoxins \\
\hline foxm1 & - & -2.03 & Oxidative stress responsive gene & prdx2 & - & -2.6143 & Peroxiredoxins \\
\hline fth1a & 2.025 & - & Oxidative stress responsive gene & prdx4 & - & -2.828 & Peroxiredoxins \\
\hline fth1b & - & -3.882 & Oxidative stress responsive gene & aox1 & - & -2.5754 & ROS Metabolism Gene \\
\hline gclm & 2.4447 & 2.5867 & Oxidative stress responsive gene & bnip3 & - & -2.3317 & ROS Metabolism Gene \\
\hline gpx1a & 2.028 & - & Oxidative stress responsive gene & sod1 & - & -2.6518 & Superoxide dismutase \\
\hline
\end{tabular}

Results are expressed as fold change (FC). Significantly expressed genes $(p<0.05)$ are highlighted in red (up-regulated), or blue (downregulated). Unchanged genes are highlighted in yellow. Gene names are the same as reported in Table 1

expressed in $\mathrm{P} 1,46$ in $\mathrm{P} 2$, and 55 in $\mathrm{P} 3$ (Table 3). The increasing number of affected genes also correlated with the increased severity of the phenotypes. The solute carrier family 25 (Slc25) proteins are carriers that facilitate the transport of solutes across the inner mitochondrial membrane. Specifically, slc25a12 (up-regulated in P2 and P3), slc25a23a (up-regulated in P3), and slc25a25 (up-regulated in all three phenotypes) are $\mathrm{Ca}^{2+}$-sensitive mitochondrial carriers (Gutiérrez-Aguilar and Baines 2013). In addition, slc25a17, up-regulated in $\mathrm{P} 2$ and $\mathrm{P} 3$, has been linked to a myopathy involving inhibition of muscular relaxation in horses, believed to be derived from an excess of calcium triggering ATP depletion (Barrey et al. 2012). These results are consistent with the reported increase in intracellular levels of $\mathrm{Ca}^{2+}$ after NMDA receptor activation by OPs (Faria et al. 2015).

Most of the proteins related to inner (timm family) or outer (tomm family) mitochondrial membrane transport were up-regulated in P2 (10) and P3 (12), and some in P1
(5), further suggesting that mitochondrial function and transport was not only important for phenotype development, but also increased with the severity of the outcome. All apoptotic genes were up-regulated in P3. Particularly, genes related to the pro-apoptotic $\mathrm{Bcl}-2$ protein $(b b c 3$, $b c l 2, b c l 21 \mathrm{l}$, and bnip3), involved in the apoptosis route (Fig. 2b) mediated by cytochrome c (Orrenius et al. 2007). This arguably indicates the potential for apoptosis leading to cell death in P3 and, in a lower amount, in P2.

In conclusion, we analyzed the expression of genes related to oxidative stress and mitochondrial function in the zebrafish model for mild, moderate, and severe OP poisoning. Our data showed that the number of genes affected increased in accordance with the severity of the phenotype. Furthermore, temporal data strongly suggest that the activation of reactive nitrogen species and peroxynitrite leading to lipid peroxidation and apoptosis might be the initial step leading to irreversibility in P3, as well as an important part of the transition from $\mathrm{P} 2$ to $\mathrm{P} 3$. 
Table 3 RT-PCR results for the P1 (Grade 1), P2 (Grade 2) and P3 (Grade 3) embryos at $24 \mathrm{~h}$ for genes related to mitochondrial function (Color table online)

\begin{tabular}{|c|c|c|c|c|c|c|c|c|c|}
\hline Symbol & P1 FC & P2 FC & P3 FC & Functional Group & Symbol & P1 FC & P2 FC & P3 FC & Functional Group \\
\hline bbc3 & - & 2.1174 & 2.8124 & Apoptotic genes & hspd1 & 6.1304 & 6.5743 & 8.5538 & Mitochondrial transport \\
\hline $\mathrm{bcl} 2$ & - & 3.0534 & 3.9475 & Apoptotic genes & immp1l & - & - & 2.2352 & Mitochondrial transport \\
\hline$b c|2| 1$ & - & - & 2.7574 & Apoptotic genes & $m t \times 2$ & - & 3.4768 & 3.5355 & Mitochondrial transport \\
\hline bida & - & - & 2.7511 & Apoptotic genes & stard3 & - & - & 3.6896 & Mitochondrial transport \\
\hline bnip3 & -2.1845 & - & 2.4371 & Apoptotic genes & timm10b & - & 2.0463 & 2.482 & Mitochondrial transport \\
\hline dnm1l & - & 3.1743 & 3.4086 & Apoptotic genes & ucp2 & 3.4586 & - & 5.6615 & Mitochondrial transport \\
\hline aifm2 & 2.4306 & 3.9653 & 4.0841 & Apoptotic genes & uср3 & 2.6183 & - & 2.9677 & Mitochondrial transport \\
\hline pmaip1 & $\cdot$ & -2.1239 & 2.2861 & Apoptotic genes & mipep & $\cdot$ & 3.2053 & 2.7571 & Mitochondrion protein import \\
\hline timm22 & 2.0673 & - & 3.3924 & Inner membrane translocation & tspo & 2.9504 & 2.1084 & 2.8329 & Mitochondrion protein import \\
\hline timm10 & $\cdot$ & 4.1991 & 2.4193 & Inner membrane translocation & tomm22 & $\cdot$ & 2.5024 & 3.9943 & Outer membrane translocation \\
\hline timm17a & 2.2366 & 2.2266 & 3.7304 & Inner membrane translocation & tomm34 & 2.9855 & 2.0165 & 4.2374 & Outer membrane translocation \\
\hline timm23 & - & - & 2.1619 & Inner membrane translocation & tomm40 & - & 2.7432 & 2.5851 & Outer membrane translocation \\
\hline timm44 & 2.2347 & 5.6039 & 6.6622 & Inner membrane translocation & tomm40I & 2.0697 & - & 2.8529 & Outer membrane translocation \\
\hline timm50 & 2.073 & 2.1084 & 2.6015 & Inner membrane translocation & tomm70a & - & - & 2.2647 & Outer membrane translocation \\
\hline timm8b & - & 2.4907 & 2.4392 & Inner membrane translocation & slc25a23a & - & - & 3.263 & Small molecule transport \\
\hline timm9 & - & 2.0964 & - & Inner membrane translocation & slc25a15b & 2.999 & - & 5.1025 & Small molecule transport \\
\hline tp53 & 2.3855 & 3.1253 & 3.7297 & Membrane Polarization & slc25a12 & - & 3.6169 & 3.1046 & Small molecule transport \\
\hline $\cos 10$ & $\cdot$ & 4.6149 & 3.7371 & Mitochondrial Fission \& Fusion & slc25a14 & - & 2.2173 & 2.1992 & Small molecule transport \\
\hline $\mathrm{mfn} 1 \mathrm{~b}$ & - & 3.2324 & 2.7763 & Mitochondrial Fission \& Fusion & slc25a15a & 14.2857 & 2.0317 & 10.5463 & Small molecule transport \\
\hline mfn2 & - & 2.0122 & 2.2019 & Mitochondrial Fission \& Fusion & slc25a16 & - & 2.2118 & 2.6601 & Small molecule transport \\
\hline Irppre & $\cdot$ & 5.1607 & 3.6227 & Mitochondrial localization & slc25a17 & $\cdot$ & 2.2365 & 2.1097 & Small molecule transport \\
\hline msto1 & - & 2.4057 & 2.0951 & Mitochondrial localization & slc25a 18 & - & 2.1829 & 3.2674 & Small molecule transport \\
\hline nefla & -10.4514 & 2.1221 & - & Mitochondrial localization & slc25a20 & - & 2.448 & - & Small molecule transport \\
\hline neflb & -3.5055 & 2.6146 & - & Mitochondrial localization & slc25a21 & - & 3.1832 & 2.7055 & Small molecule transport \\
\hline opa1 & - & 4.0381 & 3.9649 & Mitochondrial localization & slc25a22 & 2.0348 & $\cdot$ & 3.3085 & Small molecule transport \\
\hline rhot1a & $\cdot$ & 2.076 & 2.4477 & Mitochondrial localization & slc25a25b & 2.1874 & 2.1169 & 2.9754 & Small molecule transport \\
\hline cpt1b & -2.7959 & 2.8132 & 2.0235 & Mitochondrial transport & slc25a37 & 2,3599 & 3,1985 & 2.1689 & Small molecule transport \\
\hline cpt2 & - & 4.5447 & 2.198 & Mitochondrial transport & slc25a37 & 3.2402 & - & 2.7596 & Small molecule transport \\
\hline grpel1 & 2.1765 & 2.1099 & 2.652 & Mitochondrial transport & slc25a3a & -3.5781 & 3.873 & - & Small molecule transport \\
\hline hsp90aa1.2 & 16.5364 & 3.8473 & 7.9863 & Mitochondrial transport & slc25a3b & - & 2.6052 & 2.0285 & Small molecule transport \\
\hline
\end{tabular}

Results are expressed as fold change (FC). Significantly expressed genes $(p<0.05)$ are highlighted in red (up-regulated), or blue (downregulated). Unchanged genes are highlighted in yellow. Gene list: BCL2 binding component 3 (bbc3), B cell leukemia/lymphoma 2 (bcl2), Bcl2like 1 (bcl2l1), BH3 interacting domain death agonist (bida), BCL2/adenovirus E1B interacting protein 3 (bnip3), COX10 heme A:farnesyltransferase cytochrome c oxidase assembly factor (cox10), carnitine palmitoyltransferase 1B (cpt1b), carnitine palmitoyltransferase II (cpt2), dynamin 1-like (dnml l), GrpE-like 1 (grpel1), heat shock protein 90-alpha 2 (hsp90aa1.2), heat shock 60kD protein 1 (hspdl), IMP1 inner mitochondrial membrane peptidase-like (immpl l), translocase of inner mitochondrial membrane 22 (timm22), apoptosis-inducing factor mitochondrion-associated 2 (aifm2), solute carrier family 25 member 23a (slc25a23a), solute carrier family 25 member $15 \mathrm{~b}$ (slc25a15b), leucine-rich pentatricopeptide repeat containing (lrpprc), mitofusin $1 \mathrm{~b}(\mathrm{mfn} 1 \mathrm{~b})$, mitofusin 2 ( $m f n 2)$, mitochondrial intermediate peptidase (mipep), misato homolog 1 (msto1), metaxin 2 ( $m t x 2$ ), neurofilament light polypeptide-like a (nefla), neurofilament light polypeptide-like (neflb), optic atrophy 1 (opa1), phorbol-12-myristate-13-acetate-induced protein 1 (pmaip1), Ras homolog gene family, member T1a (rhotla), solute carrier family 25 member 12 (slc25a12), solute carrier family 25 member 14 (slc25a14), solute carrier family 25 member 15 a (slc25a15a), solute carrier family 25 member 16 (slc25a16), solute carrier family 25 member 17 (slc25a17), solute carrier family 25 member 18 (slc25al8), solute carrier family 25 member 20 (slc25a20), solute carrier family 25 member 21 (slc25a21), solute carrier family 25 member 22 (slc25a22), solute carrier family 25 member 25b (slc25a25b), solute carrier family 25 member 27 (slc25a27), solute carrier family 25 member 37 (slc25a37), solute carrier family 25 member 3a (slc25a3a), solute carrier family 25 member 3b (slc25a3b), START domain containing 3 (stard3), translocase of inner mitochondrial membrane 10 (timm10), translocase of inner mitochondrial membrane 10b (timm10b), translocase of inner mitochondrial membrane 17a (tim$m 17 a$ ), translocase of inner mitochondrial membrane 23 (timm23), translocase of inner mitochondrial membrane 44 (timm44), translocase of inner mitochondrial membrane 50 (timm50), translocase of inner mitochondrial membrane $8 \mathrm{~b}$ (timm $8 \mathrm{~b}$ ), translocase of inner mitochondrial membrane 9 (timm 9 ), translocase of outer mitochondrial membrane 22 (tomm22), translocase of outer mitochondrial membrane 34 (tomm34), translocase of outer mitochondrial membrane 40 (tomm 40), translocase of outer mitochondrial membrane 40 like (tomm $40 l$ ), translocase of outer mitochondrial membrane 70a (tomm70a), tumor protein p53 (tp53), translocator protein (tspo), uncoupling protein 2 (ucp2), uncoupling protein 3 (ucp3) 
Acknowledgments This work was supported in part by the US Army ERDC-IRO (W912HZ-13-BAA-01; D.R., N.G.R.) and Environmental Quality Research Program (N.G.R.), the NATO SfP project MD.SFPP 984777 (D.R., N.G.R.), and the National Science Foundation EPSCoR Grant EPS-0903787 (N.G.R.).

Open Access This article is distributed under the terms of the Creative Commons Attribution 4.0 International License (http://crea tivecommons.org/licenses/by/4.0/), which permits unrestricted use, distribution, and reproduction in any medium, provided you give appropriate credit to the original author(s) and the source, provide a link to the Creative Commons license, and indicate if changes were made.

\section{References}

Barrey E, Jayr L, Mucher E et al (2012) Transcriptome analysis of muscle in horses suffering from recurrent exertional rhabdomyolysis revealed energetic pathway alterations and disruption in the cytosolic calcium regulation. Anim Genet 43:271-281

Bertolote JM (2006) Deaths from pesticide poisoning: a global response. Br J Psychiatry 189:201-203. doi:10.1192/bjp.bp.105. 020834

Brookes PS (2004) Calcium, ATP, and ROS: a mitochondrial lovehate triangle. AJP Cell Physiol 287:C817-C833

Calatayud-Vernich P, Calatayud F, Simó E et al (2016) Influence of pesticide use in fruit orchards during blooming on honeybee mortality in 4 experimental apiaries. Sci Total Environ 541:33-41

Dong X-X, Wang Y, Qin Z-H (2009) Molecular mechanisms of excitotoxicity and their relevance to pathogenesis of neurodegenerative diseases. Acta Pharmacol Sin 30:379-387

Echeverría-Sáenz S, Mena F, Pinnock M et al (2012) Environmental hazards of pesticides from pineapple crop production in the Río Jiménez watershed (Caribbean Coast, Costa Rica). Sci Total Environ 440:106-114

Faria M, Garcia-Reyero N, Padrós F et al (2015) Zebrafish models for human acute organophosphorus ooisoning. Sci Rep. doi:10.1038/ srep15591

Gutiérrez-Aguilar M, Baines CP (2013) Physiological and pathological roles of mitochondrial SLC25 carriers: figure 1. Biochem J 454:371-386

Jacobson SM, Birkholz DA, McNamara ML, Bharate SB, George KM (2010) Subacute developmental exposure of zebrafish to the organophosphate pesticide metabolite, chlorpyrifos-oxon, results in defects in Rohon-Beard sensory neuron development. Aquat Toxicol 100(1):101-111. doi:10.1016/j.aquatox.2010.07.015

Kaur S, Singh S, Singh C, Prakash A (2014) Potential pharmacological strategies for the improved treatment of organophosphateinduced neurotoxicity. Can J Physiol Pharmacol 92(11):893-911

Kritis AA, Stamoula EG, Paniskaki KA, Vavilis TD (2015) Researching glutamate â€"induced cytotoxicity in different cell lines: a comparative/collective analysis/study. Front Cell Neurosci 9:510. doi:10.1016/j.biochi.2011.09.013

Macneale KH, Spromberg JA, Baldwin DH, Scholz NL (2014) A Modeled comparison of direct and food web-mediated impacts of common pesticides on Pacific salmon. PLoS One 9:e92436. doi:10.1371/journal.pone.0092436.s002

Mei W, Peng Z, Lu M et al (2015) Peroxiredoxin 1 inhibits the oxidative stress induced apoptosis in renal tubulointerstitial fibrosis. Nephrology (Carlton) 20:832-842. doi:10.1111/nep. 12515

Moncada S, Bolanos JP (2006) Nitric oxide, cell bioenergetics and neurodegeneration. J Neurochem 97:1676-1689

Orrenius S, Gogvadze V, Zhivotovsky B (2007) Mitochondrial oxidative stress: implications for cell death. Annu Rev Pharmacol Toxicol 47:143-183

Orrenius S, Gogvadze V, Zhivotovsky B (2015) Biochemical and biophysical research communications. Biochem Biophys Res Commun 460:72-81. doi:10.1016/j.bbrc.2015.01.137

Pena-Llopis S (2005) Antioxidants as potentially safe antidotes for organophosphorus poisoning. Curr Enzyme Inhib 1:147-156. doi: $10.2174 / 1573408054022243$

Rizzuto R, De Stefani D, Raffaello A, Mammucari C (2012) Mitochondria as sensors and regulators of calcium signalling. Nat Rev Mol Cell Biol 13:566-578

Southam A, Lange A, Hines A et al (2011) Metabolomics reveals target and off-Target toxicities of a model organophosphate pesticide to roach (Rutilus rutilus): implications for biomonitoring. Environ Sci Tecnol 45(8):3759-3767

Trujillo M, Ferrer-Sueta G, Radi R (2008) Kinetic studies on peroxynitrite reduction by peroxiredoxins. Methods Enzymol 441:173-196

Zhu W, Schmehl DR, Mullin CA, Frazier JL (2014) Four common pesticides, their mixtures and a formulation solvent in the hive environment have high oral toxicity to honey bee larvae. PLoS One 9:e77547. doi:10.1371/journal.pone.0077547 